\title{
Hysterectomy trends for benign indications over a 15-year period in an academic teaching center in Poland: a retrospective cohort study
}

\author{
Katarzyna Romanek-Piva, Krzysztof Gałczyński, Aneta Adamiak-Godlewska, Konrad Futyma, \\ Paweł Miotła, Tomasz Rechberger
}

$2^{\text {nd }}$ Department of Gynecology, Medical University of Lublin, Poland

\begin{abstract}
Objectives: The aim of the study was to evaluate changes in the operative trends for various types of hysterectomy due to benign indications, between 2001 and 2015, at the $2^{\text {nd }}$ Department of Gynecology, Medical University of Lublin, as compared to the National Health Service (NHS) registry in Poland.

Material and methods: A retrospective cohort study was conducted. Data from the Internal Hospital Discharge Registry and Pathological Results Registry have been compared to the NHS database, which has been available nationwide since 2009.

Results: The study group included 5629 women who underwent hysterectomy due to benign indications. During the study period, the following number of procedures were performed: total abdominal hysterectomy $-344(6.11 \%)$, total abdominal hysterectomy with bilateral salpingo-oophorectomy -1760 (31.27\%), total vaginal hysterectomy $-563(10.00 \%)$, subtotal abdominal hysterectomy - 2536 (45.05\%), and laparoscopically-assisted subtotal hysterectomy (LASH) - 426 (7.57\%). The abdominal route, with the preference for subtotal abdominal hysterectomy, was the main approach to hysterectomy. Symptomatic fibroids were the most common indication for the procedure. Comparison of data collected over the last five years revealed a significant difference in the approach to hysterectomy in favor of subtotal abdominal hysterectomy (SAH) and LASH.

Conclusions: Less invasive techniques of hysterectomy (LASH, SAH), which are the preferred choice at the $2^{\text {nd }}$ Department of Gynecology (Lublin), are safe and effective options of treating benign conditions. We are of the opinion that these approaches should be offered to patients instead of more radical techniques. Proper training of physicians may influence the decision-making process in favor of minimally invasive techniques.
\end{abstract}

Key words: laparoscopy, hysterectomy, operative tends

Ginekologia Polska 2016; 87, 6: 411-416

\section{INTRODUCTION}

Hysterectomy following a cesarean section is the second most common gynecological surgery in the world [1]. It is essential to collect information about the main indications, outcome, and postoperative quality of patient life, as it could modify the clinical approach to this procedure in the future. According to a recent report from the Organization for Economic Co-operation and Development (OECD) on 'International variations in a selected number of surgical procedures' among OECD countries, a statistically significant decrease in hysterectomy rates has been reported in New Zealand, Australia, Canada, and the USA. However, such a trend has not been observed in Europe. Although the rates vary between and within countries, it has been estimated that approximately 5.5 hysterectomies per 1000 women are performed in the USA, 3.3/1000 in Australia, 1.14/1000 in Mexico, 4.2/1000 in the United Kingdom, 5.26/1000 in Norway, and 5.0/1000 in Poland, annually. Uterine fibroids, abnormal uterine bleeding (AUB), pelvic organ prolapse (POP), and endometriosis constitute over $90 \%$ of all surgical indications for hysterectomy [1-4]. In the past, other benign conditions like ovarian cysts and pelvic 
pain were also considered as indications for uterine removal. However, hysterectomy rates have been slowly decreasing recently due to the availability of less invasive techniques for treating myomas or abnormal uterine bleeding, including endoscopy procedures, arterial embolization, hormonal therapy, and percutaneous laser thermoablation [5]. In some cases, emergency hysterectomy must be performed due to postpartum hemorrhage, pyometra with no response to antibiotic treatment, and excessive uterine bleeding with no success of other therapeutic options [6].

Traditionally, hysterectomy has been performed via the abdominal route (total — TAH), with or without bilateral salpingo-oophorectomy (TAH/BSO), and subtotal abdominal hysterectomy $(\mathrm{SAH})$, or via the vaginal routes: total vaginal hysterectomy $(\mathrm{TVH})$ or laparoscopically-assisted vaginal hysterectomy (LAVH), or subtotal hysterectomy (LASH) (Figure 1). Recent data have suggested a steady increase in hysterectomies using minimal-access techniques (e.g. total or subtotal laparoscopic hysterectomy performed entirely via the laparoscopic route). It is one of the most complex types of laparoscopic surgical approaches in gynecology, requiring the highest level of surgical expertise [7]. At present, most hysterectomies worldwide are performed via the abdominal route, despite numerous recommendations for the main surgical approach to hysterectomy to be vaginal or laparoscopic. Vaginal hysterectomy $(\mathrm{VH})$ is recommended as the first choice and should be preferred to abdominal hysterectomy $(\mathrm{AH})$. Other surgical options, including $\mathrm{AH}$ and laparoscopic hysterectomy, should be considered only in cases when $\mathrm{VH}$ is not technically possible. Although it has been well-documented that the vaginal or laparoscopic approaches are connected with less postoperative pain and fewer complications, more rapid recovery in a hospital, less blood loss, and less cost, approximately $60 \%$ of the procedures continue to be performed abdominally. Variations in the surgical techniques have been introduced to reduce operative morbidity and postoperative effects of hysterectomy on the urinary and sexual functions [8-11].
Since 2001, many studies have demonstrated that the Fallopian tube might be the origin of most high-grade ovarian and peritoneal serous carcinomas. Simple modifications in the surgical practice, i.e. prophylactic bilateral salpingectomy, could significantly decrease future mortality rates due to ovarian cancer $[11,12]$. In light of these publications, in April 2011, our center introduced prophylactic bilateral salpingectomy with hysterectomies performed via the abdominal and laparoscopic routes. Nevertheless, substantial variations in the preferred mode of elective hysterectomy continue to persist $[4,13-16]$.

\section{OBJECTIVES}

The aim of the study was to analyze the changing trends in surgical approach to hysterectomy at the $2^{\text {nd }}$ Department of Gynecology, Medical University of Lublin, between 2001 and 2015, in comparison to the nationwide trend analysis of the National Health Services (NHS) database. These findings aim to encourage further enquiry and broaden our understanding of the need for appropriate guidelines and focus on adequate gynecological training.

\section{MATERIAL AND METHODS}

The NHS Registry in Poland for operative procedures in gynecology was established in 2009. No earlier national combined data have been available. For that reason, a retrospective study based on the hospital registry of internal surgical interventions and postoperative histological results of patients who underwent hysterectomy at the $2^{\text {nd }}$ Department of Gynecology, Medical University of Lublin (Poland), from January 1, 2001 to December 31, 2015, has been performed. A form was designed especially for the purpose of the study and included the following information: dates of hospital admission and discharge, main diagnosis, and type of surgical intervention, recorded according to the International Classification of Disease (ICD-10 and ICD-9). Indications for hysterectomy were determined using the ICD-9 and ICD-10 nomenclature and grouped into: menor-

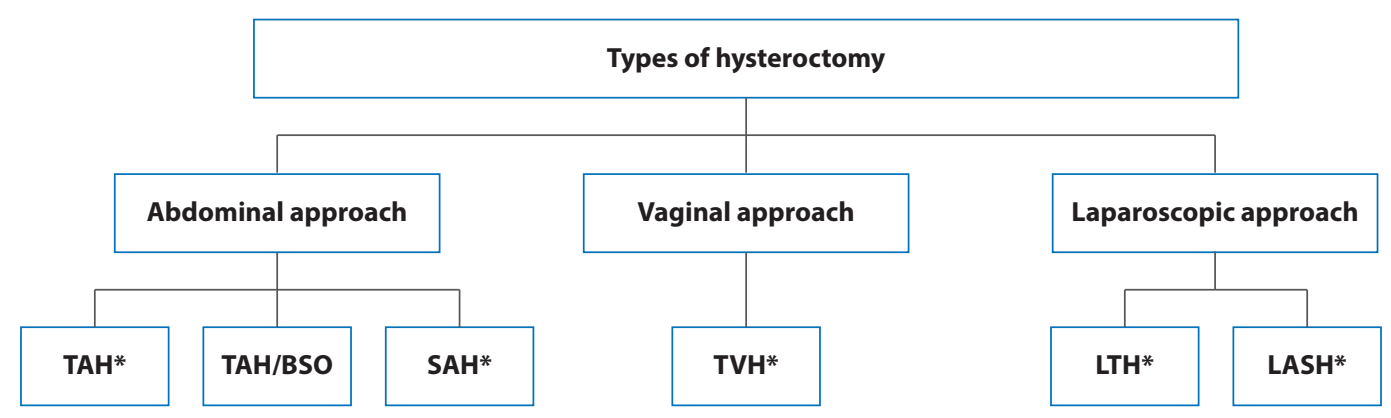

Figure 1. Types of hysterectomy. *Since 2011 , all hysterectomy types at the $2^{\text {nd }}$ Department of Gynecology have been performed together with bilateral salpingectomies; TAH — total abdominal hysterectomy; TAH/BSO — total abdominal hysterectomy with bilateral salpingo-oophorectomy; SAH — subtotal abdominal hysterectomy; TVH — total vaginal hysterectomy; LTH — laparoscopically-assisted hysterectomy; LASH — laparoscopically-assisted subtotal hysterectomy 
rhagia, pelvic organ prolapse, uterine fibroids, endometriosis, adenomyosis, endometrial hyperplasia, pelvic pain, and benign ovarian cyst. A total of 6563 cases of hysterectomies were analyzed and further classified into 5 groups according to surgery type: TAH, TAH with unilateral or bilateral salpingo-oophorectomy - both groups were included into the 'TAH/BSO' category due to highly detailed data, $\mathrm{TVH}$, SAH (since 2011 combined with prophylactic salpingectomy at our Department), and LASH. For the purpose of this study, 934 cases of hysterectomies due to malignant tumors recognized in histopathological exam results have been excluded. We compared our data and results with the Polish national trends using the NHS database, which contains information on patients admitted to all public hospitals in Poland [15].

\section{Data analysis}

Data were analyzed using Microsoft Excel 2010. As stated above, the Internal Hospital Registry covers all cases of hysterectomies performed at the $2^{\text {nd }}$ Department of Gynecology, Medical University of Lublin, between 2001 and 2015. Since 2009, the NHS Registry database have included data on all hysterectomy procedures performed in Poland annually.

\section{RESULTS}

A total of 6563 women who underwent various types of hysterectomy were identified. Between 2001 and 2015, a total number of 5629 hysterectomies due to benign conditions were performed at the $2^{\text {nd }}$ Department of Gynecology. The main indications for hysterectomy (Table 1) with annual differences between them (Figure 2) and the number of various types of procedures (Table 2 ) are presented below.

Between 2001 and 2015, the main approach to hysterectomy was the abdominal route, with preference for SAH, followed by TAH with bilateral salpingo-oophorectomy ( 2536 vs. 1760 cases; $45.05 \%$ vs. $31.27 \%$, respectively). Since 2007 , the number of TAH/BSO decreased by $49.8 \%$ and the number of SAH increased by $46.81 \%$, as compared to the period between 2001 and 2006. During the study period, no important changes in the TVH rate have been reported. Since the beginning of 2011, a notable increase in the number of LASH has been observed.

According to NHS data on hysterectomies performed in Poland between 2009-2014, mean rates of TAH, TAH/BSO, TVH, SAH and LASH were 35.2\%, 17.9\%, 2.8\%, 21\% and 2.14\%, respectively. During the same time interval, mean rates of TAH, TAH/BSO, TVH, SAH and LASH at the $2^{\text {nd }}$ Department of Gynecology were $5.5 \%, 17.1 \%, 6.3 \%, 58.5$ and $12.5 \%$, respec-

\begin{tabular}{|c|c|c|c|c|c|c|c|c|c|c|}
\hline \multirow{3}{*}{ Year } & \multicolumn{10}{|c|}{ Indications } \\
\hline & \multicolumn{2}{|c|}{ Fibroids } & \multicolumn{2}{|c|}{ AUB } & \multicolumn{2}{|c|}{ POP } & \multicolumn{2}{|c|}{ Endometriosis } & \multicolumn{2}{|c|}{ Other } \\
\hline & $\mathbf{n}$ & $(\%)$ & $\mathbf{n}$ & (\%) & $\mathbf{n}$ & $(\%)$ & $\mathbf{n}$ & (\%) & n & (\%) \\
\hline 2001 & 171 & 62.1 & 27 & 9.8 & 46 & 16.8 & 11 & 4.0 & 20 & 7.3 \\
\hline 2002 & 146 & 49.3 & 13 & 4.3 & 71 & 24.0 & 23 & 7.8 & 43 & 14.5 \\
\hline 2003 & 168 & 60.4 & 20 & 7.2 & 42 & 15.1 & 17 & 6.1 & 31 & 11.2 \\
\hline 2004 & 201 & 55.2 & 24 & 6.6 & 64 & 17.6 & 29 & 7.8 & 46 & 12.6 \\
\hline 2005 & 156 & 53.2 & 24 & 8.2 & 41 & 14.0 & 21 & 7.2 & 51 & 17.4 \\
\hline 2006 & 181 & 52.3 & 31 & 8.9 & 27 & 7.8 & 36 & 10.4 & 71 & 20.5 \\
\hline 2007 & 216 & 52.2 & 52 & 12.6 & 32 & 7.7 & 25 & 6.0 & 89 & 21.5 \\
\hline 2008 & 211 & 55.7 & 37 & 9.76 & 23 & 6.1 & 11 & 2.9 & 97 & 25.6 \\
\hline 2009 & 289 & 61.4 & 42 & 8.9 & 15 & 3.2 & 29 & 6.2 & 96 & 20.4 \\
\hline 2010 & 263 & 61.0 & 27 & 6.3 & 21 & 4.9 & 41 & 9.5 & 79 & 18.3 \\
\hline 2011 & 301 & 60.4 & 48 & 9.6 & 13 & 2.6 & 35 & 7.0 & 101 & 20.3 \\
\hline 2012 & 276 & 56.8 & 67 & 13.8 & 11 & 2.3 & 44 & 9.0 & 88 & 18.1 \\
\hline 2013 & 267 & 65.0 & 38 & 9.3 & 16 & 3.9 & 29 & 7.1 & 61 & 14.8 \\
\hline 2014 & 246 & 66.5 & 34 & 9.2 & 9 & 2.4 & 8 & 2.2 & 73 & 19.7 \\
\hline 2015 & 221 & 69.7 & 38 & 12.0 & 13 & 4.1 & 6 & 1.9 & 39 & 12.3 \\
\hline Total number of procedures & 3313 & 58.9 & 522 & 9.3 & 444 & 7.8 & 365 & 6.5 & 985 & 17.5 \\
\hline
\end{tabular}

AUB — abnormal uterine bleeding; POP — pelvic organ prolapse 


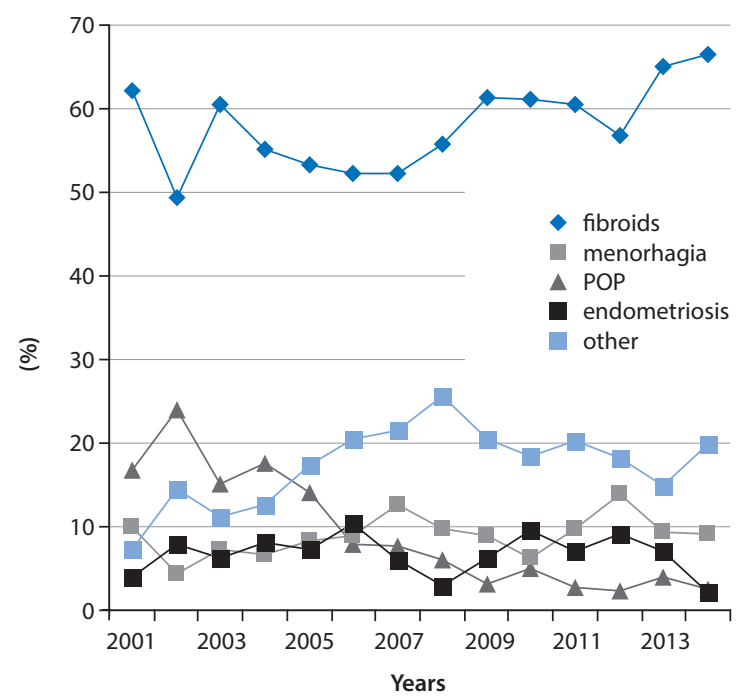

Figure 2. Annual differences between the main indications for hysterectomy. The presence of symptomatic fibroids, followed by abnormal uterine bleeding (AUB), pelvic organ prolapse (POP), endometriosis and other causes (adenomyosis, endometrial hyperplasia, benign ovarian cysts), constituted the most common indication for hysterectomy. The number of hysterectomies performed due to POP decreased since 2005, after the introduction of vaginal mesh procedures in our department tively. Data comparison revealed a significant difference in the approach to hysterectomy (in favor of SAH and minimally invasive techniques) at our Department. Mean TAH/BSO rates were similar but the TAH rate was higher in the NHS database as compared to our registry (Figure 3 ).

\section{DISCUSSION}

Hysterectomy remains to be the most common procedure among gynecologic surgeries. The latest studies have suggested the preferred approach to be the laparoscopic or vaginal route, if possible. The evidence in favor of minimally-invasive hysterectomies is reflected in the current international guidelines and is associated with decreased perioperative morbidity $[17,18]$. The introduction of laparoscopy into gynecological surgical practice has changed hysterectomy trends dramatically [18]. The proportion of hysterectomies performed laparoscopically in the USA has increased significantly during the last 20 years, from $0.3 \%$ in 1990 to $11.8 \%$ in 2003 [19]. Recently, FDA issued a statement concerning the safety of power morcellation in case of LASH, especially in terms of oncological risks [20]. The most recent analyses have clearly indicated that power morcellation is safe, provided indications for such procedures are followed $[21,22]$. Regardless, the abdominal approach continues

\begin{tabular}{|c|c|c|c|c|c|c|c|}
\hline \multirow{2}{*}{ Year } & & \multicolumn{6}{|c|}{ Type of surgery } \\
\hline & & TAH & TAH/BSO & TVH & SAH & LASH & $\begin{array}{l}\text { Total no of } \\
\text { procedures }\end{array}$ \\
\hline \multicolumn{2}{|l|}{2001} & 18 & 194 & 51 & 12 & 0 & 275 \\
\hline \multicolumn{2}{|l|}{2002} & 17 & 228 & 44 & 7 & 0 & 296 \\
\hline \multicolumn{2}{|l|}{2003} & 15 & 170 & 38 & 55 & 0 & 278 \\
\hline \multicolumn{2}{|l|}{2004} & 32 & 169 & 61 & 102 & 0 & 364 \\
\hline \multicolumn{2}{|l|}{2005} & 20 & 137 & 53 & 83 & 0 & 293 \\
\hline \multicolumn{2}{|l|}{2006} & 36 & 170 & 20 & 120 & 0 & 346 \\
\hline \multicolumn{2}{|l|}{2007} & 24 & 74 & 39 & 277 & 0 & 414 \\
\hline \multicolumn{2}{|l|}{2008} & 12 & 122 & 56 & 189 & 0 & 379 \\
\hline \multicolumn{2}{|l|}{2009} & 21 & 117 & 14 & 319 & 0 & 471 \\
\hline \multicolumn{2}{|l|}{2010} & 19 & 99 & 27 & 286 & 0 & 431 \\
\hline \multicolumn{2}{|l|}{2011} & 17 & 74 & 34 & 365 & 8 & 498 \\
\hline \multicolumn{2}{|l|}{2012} & 13 & 53 & 51 & 338 & 31 & 486 \\
\hline \multicolumn{2}{|l|}{2013} & 29 & 46 & 27 & 163 & 146 & 411 \\
\hline \multicolumn{2}{|l|}{2014} & 41 & 66 & 19 & 127 & 117 & 370 \\
\hline \multicolumn{2}{|l|}{2015} & 30 & 41 & 29 & 93 & 124 & 317 \\
\hline \multirow{2}{*}{ Total number } & $n$ & 344 & 1760 & 563 & 2536 & 426 & 5629 \\
\hline & (\%) & 6.11 & 31.27 & 10.00 & 45.05 & 7.57 & 100 \\
\hline
\end{tabular}

$\mathrm{TAH}$ - total abdominal hysterectomy; TAH/BSO — total abdominal hysterectomy with bilateral salpingo-oophorectomy; TVH — total vaginal hysterectomy; SAH — subtotal abdominal hysterectomy; LASH — laparoscopically-assisted subtotal hysterectomy 


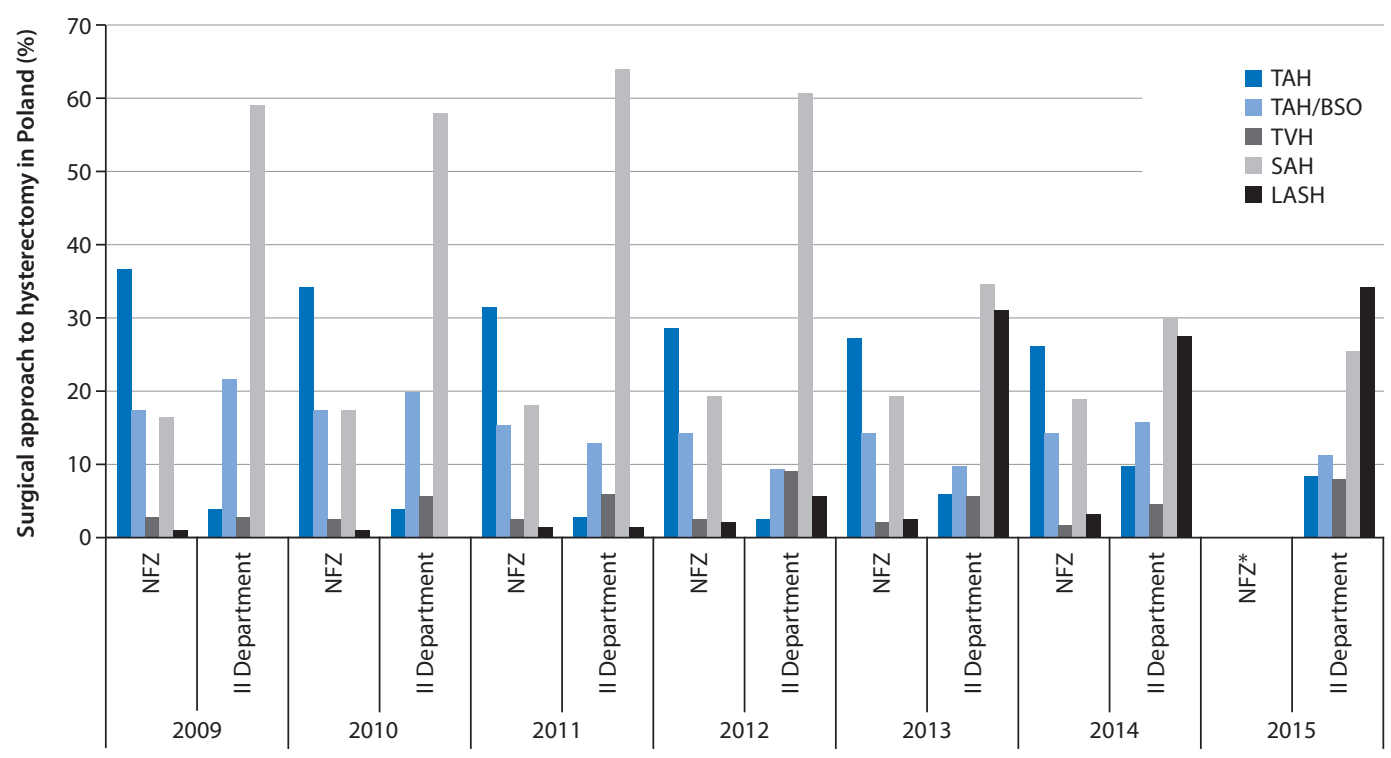

Figure 3. Comparison of national NHS database and internal trends for hysterectomy between 2009 and 2015 (no NHS data were available for the period between 2001-2008); NFZ — Narodowy Fundusz Zdrowia; TAH — total abdominal hysterectomy; TAH/BSO — total abdominal hysterectomy with bilateral salpingo-oophorectomy; TVH — total vaginal hysterectomy; $\mathrm{SAH}$ — subtotal abdominal hysterectomy; LASH — laparoscopically-assisted subtotal hysterectomy

to be the mostly common choice worldwide. To the best of our knowledge, our cross-sectional study has been the first to compare different types of hysterectomy in Poland both, locally and nationwide. The investigated trends in methods of uterine removal and the number of procedures have remained stable over the last 15 years of observation, but several significant changes in operative trends for hysterectomy have been noticed at the $2^{\text {nd }}$ Department of Gynecology, Medical University of Lublin.

The analysis of SAH and LASH rates has clearly demonstrated a difference between NHS data and our Department. The number of SAH and LASH procedures in our department was almost 3- and 6-fold higher, respectively as compared to data from Poland. New international trends to abandon total radical hysterectomy in favor of more harmless procedures like laparoscopic-assisted hysterectomy or subtotal hysterectomy have been discussed in several gynecological congresses and publications. Due to lack of well-defined indications for subtotal hysterectomy, the final decision is made by the surgeon and based on patient condition and wishes. Total abdominal hysterectomy, unless necessary, can cause sexual dysfunction due to shortened vaginal cuff, increased morbidity, prolonged time of operation, higher blood loss, increased risk of pelvic organ prolapse, and complication in wound healing. In our opinion, this less- or minimally-invasive approach to hysterectomy should be first taken into account before applying more radical procedures. However, the benefits from minimally-invasive surgery are weighed against the reported increase in urologic injury during laparoscopic hysterectomy [8].
No cases of laparoscopic hysterectomies reported at the $2^{\text {nd }}$ Department of Gynecology and very low numbers reported in Poland between 2001-2010 can be explained by the lack of necessary equipment in the operating room, financial and NHS refund issues, as well as lack of proper training. The recent increase in minimally-invasive hysterectomies may be reflected in cost-effectiveness i.e.: shorter recovery time, less blood loss, fewer infections, as well as more exposure to laparoscopy training. Furthermore, prophylactic salpingectomies for the prevention of ovarian cancer have been performed at our Department since 2010. The functional and anatomical outcome, quality of life and sexual function of patients who underwent hysterectomy for benign conditions has been published elsewhere $[23,24]$. We are aware of the fact that several factors can be associated with different choice of hysterectomy type, including geographic location, race and ethnicity, hospital grade, operating room equipment, and training pattern, while NHS reports show only the average nationwide trend in Poland $[18,19]$. This is the biggest cohort study evaluating the changing trends in operative gynecology conducted up-to-date in Poland. However, these studies have not examined factors which may influence the choice of hysterectomy type.

The ideal choice of the surgical approach to hysterectomy should be based on patient clinical presentation, experience and skill of the surgeon, evidence-based practice, and patient preferences. The surgery method should be as harmless as possible, while providing adequate treatment and optimal medical care. Although the benefits of 
minimally-invasive surgery have been well-documented, all options of surgical approach should be readily available for appropriately selected patients. Further research is needed to better understand factors influencing hysterectomy trends in different gynecology centers.

\section{CONCLUSIONS}

Less invasive techniques of hysterectomy (LASH, SAH), which are preferred at the $2^{\text {nd }}$ Department of Gynecology in Lublin, are effective and safe options for treating benign conditions. We are of the opinion that these approaches should be offered to patients instead of more radical techniques. Proper training of physicians may influence the decision-making process in favor of minimally invasive techniques.

\section{REFERENCES}

1. Carlson KJ, Nicholas D, Schiff I. Indications for hysterectomy. N Engl J Med. 1982, 328, 856-860.

2. Keshavarz H, Hillis SD, Kieke BA, [et al.]. Hysterectomy surveillance - United States, 1994-1999. MMWR CDC Surveill Summ. 2002, 51 (SS-5), 1-8.

3. Coulter A, MCPherson K, Vessey M. Do British women undergo too many or too few hysterectomies? Soc Sci Med. 1988, 27, 987-994.

4. Organization for Economic Co-operation and Development. International Variations in rates of selected surgical procedures across OEDC countries. Report October 3. 2011, 19. http://www.oecd. org/els/health-systems/48831231.pdf

5. Scialli AR. Alternatives to hysterectomy for benign conditions. Int J Fert Womens Med. 1998, 43, 186-191.

6. Lefebvre G, Allaire C, Jeffrey J, [et al.]. Hysterectomy. J Obstet Gynaecol Can. 2002, 24, 37-48.

7. Walsh CA, Walsh SR, Tang TY, [et al.]. Total abdominal hysterectomy versus total laparoscopic hysterectomy for benign disease: a meta-analysis. Eur J Obstet Gynecol Reprod Biol. 2009, 144, 3-7.
8. Johnson N, Barlow D, Lethaby A, [et al.]. Surgical approach to hysterectomy for benign gynaecological disease. The Cochrane Database Syst Rev. 2006, 19, CD003677.

9. Hanley HG. The late urological complications of total hysterectomy. $\mathrm{Br}$ J Urol. 1969, 41, 682-684.

10. Farrel SA, Kieser K. Sexuality after hysterectomy. Obstet Gynecol. 2000, 95, 1045-1051.

11. McAlpine JN, Hanley GE, Woo MM, [et al.]. Opportunistic salpingectomy: uptake, risks, and complications of a regional initiative for ovarian cancer prevention. Ovarian Cancer Research Program of British Columbia. Am J Obstet Gynecol. 2014, 210, 471.e1-471.e11.

12. Chene G, Rahimi K, Mes-Masson AM, [et al.]. Surgical implications of the potential new tubal pathway for ovarian carcinogenesis. JMinim Invasive Gynecol. 2013, 20, 153-159.

13. Nowak-Markwitz E, Spaczyński M. Ovarian cancer - modern approach to its origin and histogenesis. Ginekol Pol. 2012, 83, 454-457-170.

14. Gimbel H, Ottesen B, Tabor A. Danish gynecologists'opinion about hysterectomy on benign indication: results of a survey. Acta Obstet Gynecol Scand. 2002, 81, 1123-1131.

15. National Health Service Database (NFZ) https://prog.nfz.gov. pl/APP-JGP/KatalogJGP.aspx.

16. ACOG committee opinion no. 444: Choosing the route of hysterectomy for benign disease. Obstet Gynecol. 2009, 114, 1156-1158.

17. Jacoby VL, Autry A, Jacobson G, [et al.]. Nationawide se of laparoscopic hysterectomy compared with abdominal and vaginal approaches. Obstet Gynecol. 2009, 114, 1041-1048.

18. Wu JM, Wechter ME, Geller EJ, [et al.]. Hysterectomy rates in the United States, 2003. Obstet Gynecol. 2007, 110, 1091-1095.

19. Harris JA, Swenson CW, Uppal S, [et al.]. Practice patterns and postoperative complications before and after US Food and Drug Administration safety communication on power morcellation. Am J Obstet Gynecol. 2016, 214, 98.e1-98.e13.

20. Pritts EA, Vanness DJ, Berek JS, [et al.]. The prevalence of occult leiomyosarcoma at surgery for presumed uterine fibroids: a meta-analysis. Gynecol Surg. 2015, 12, 165-177.

21. Brölmann $H$, Tanos V, Grimbizis $G$, [et al.]. Options on fibroid morcellation: a literature review. Gynecol Surg. 2015, 12, 3-15.

22. Skorupska K, Miotła P, Kubik-Komar A, [et al.] Are there any differences in quality of life and sexual functions after various types of hysterectomy — does prophylactic salpingectomy matter? Ginekol Pol. 2016, 87, 26-31.

23. Skorupska K, Miotła P, Kubik-Komar A, [et al.] Urinary incontinence after hysterectomy — does the type of surgery matter? Ginekol Pol. 2016, 87, 94-97. 